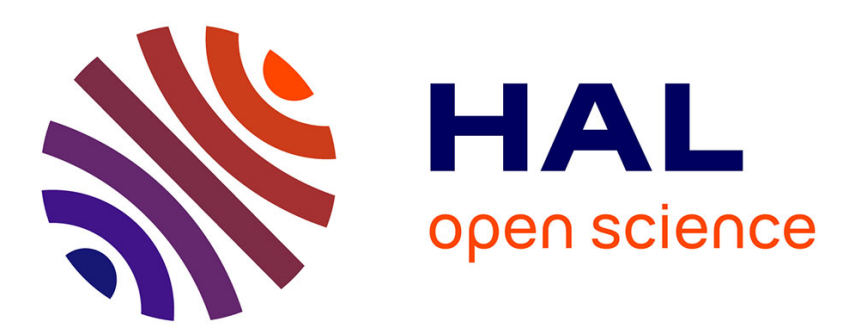

\title{
Myristoylation, an ancient protein modification mirroring eukaryogenesis and evolution
}

Thierry Meinnel, Cyril Dian, Carmela Giglione

\section{To cite this version:}

Thierry Meinnel, Cyril Dian, Carmela Giglione. Myristoylation, an ancient protein modification mirroring eukaryogenesis and evolution. Trends in Biochemical Sciences, 2020, 45 (7), pp.619-632. 10.1016/j.tibs.2020.03.007 . hal-02571405

\section{HAL Id: hal-02571405 \\ https://hal.science/hal-02571405}

Submitted on 16 Nov 2020

HAL is a multi-disciplinary open access archive for the deposit and dissemination of scientific research documents, whether they are published or not. The documents may come from teaching and research institutions in France or abroad, or from public or private research centers.
L'archive ouverte pluridisciplinaire $\mathbf{H A L}$, est destinée au dépôt et à la diffusion de documents scientifiques de niveau recherche, publiés ou non, émanant des établissements d'enseignement et de recherche français ou étrangers, des laboratoires publics ou privés. 

mirroring eukaryogenesis and evolution

13 Université Paris-Saclay, CEA, CNRS, Institute for Integrative Biology of the Cell (I2BC), 91198, Gif14 sur-Yvette, France

23 Keywords: acetylation; glycine; membrane; myristoylation; quality control; subcellular 24 compartments 


\section{Abstract}

$27 \mathrm{~N}$-myristoylation (MYR) is a crucial fatty acylation catalyzed by N-myristoyltransferases (NMTs)

28 that is likely to have appeared over two billion years ago. Proteome-wide approaches have now 29 delivered an exhaustive list of substrates undergoing MYR across approximately 2\% of any 30 proteome, with constituents, several unexpected, associated with different membrane 31 compartments. A set of $<10$ proteins conserved in eukaryotes probably represents the original 32 set of $\mathrm{N}$-myristoylated targets, marking major changes occurring throughout eukaryogenesis. 33 Recent findings have revealed unexpected mechanisms and reactivity, suggesting competition 34 with other acylations that are likely to influence cellular homeostasis and the steady state of the 35 modification landscape. Here, we review recent advances in NMT catalysis, substrate specificity, 36 and MYR proteomics, and discuss concepts regarding MYR during evolution. 
39 Plasma membranes (PMs) are composed of extrinsic and intrinsic proteins (52\%) and lipids $40(40 \%)$, the latter sustaining the overall cellular architecture. Membrane-penetrating extrinsic 41 proteins often possess covalently linked lipids, usually fatty acids, which allow the protein to 42 contact other intra- and extracellular proteins [1]. Protein lipidation involves amides (i.e. $\mathbf{N}$ - $\boldsymbol{\alpha}$ 43 myristoylation, MYR, see Glossary and glycosylphosphatidylinositol (GPI) anchors), thioesters 44 (i.e. S-palmitoylation, PAL), and thioethers (i.e. isoprenylation and farnesylation) [2]. Of these, 45 MYR is a frequent and conserved modification specific to eukaryotes that targets major cellular 46 components. Mapping the proteins undergoing MYR has proven challenging due to their difficult 47 handling characteristics and amphiphilic, chimeric nature. Recently, high-end technologies have 48 allowed the first lipidated proteome, the myristoylome, to be described in detail in various organisms recapitulating the tree of life [3]. These and other studies on myristoylome 50 composition and genesis have also revealed (i) an unexpected novel mechanism of action of $\mathbf{N}$ 51 myristoyltransferase (NMT), (ii) NMT substrate selectivity, and (iii) the capacity of NMT to act on $52 \mathrm{~N}$-terminal lysines (Lys) as well as the usual glycines (Gly).

53 This review highlights the series of ground-breaking discoveries that have recently 54 significantly advanced our knowledge about this long-studied enzyme. This includes an overview 55 of a new NMT catalytic mechanism, substrate specificity, and proteomics, and a discussion of how 56 a reduced set of targets is closely related to eukaryogenesis and eukaryote evolution.

58 How NMTs catalyze MYR with high selectivity NMTs are GNAT members closely related to No-acetyltransferases

60 Seventy-four crystal structures have now revealed that the C-terminal 400 amino acid-long 61 NMT catalytic core displays a conserved 3D GCN5-related N-acetyltransferase (GNAT) core.

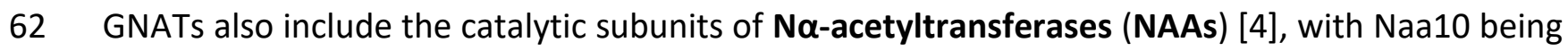
63 the closest to NMT as it modifies N-terminal Gly [5-7]. NMTs have two adjacent GNAT domains, 64 most likely to have arisen through duplication of a Naa10-like GNAT domain [8]. Prokaryotes do 65 not possess NMTs, which seem to have arisen as eukaryotes evolved from the last archaeal 
common ancestor (LACA) to the last eukaryotic common ancestor (LECA). Therefore, NMTs are

67 associated with events during eukaryogenesis.

68 How NMTs promote high substrate specificity

69 NMTs are classified as glycylpeptide N-tetradecanoyltransferases because MYR was thought 70 to only modify N-terminal Gly residues. As Gly usually arises from co-translational methionine 71 excision, the $\mathbf{N}$-myristoylated (MYRed) Gly is Gly2 (Met1 being aa1, and aax referring to the 72 following amino acids at position $x$ ). Post-translational MYR has also been reported (see below)

\section{Structural approaches}

74 Structures of human NMT1 (HsNMT1) co-crystallized with reactive substrates, including polypeptide substrates, have recently become available [7]. These peptide-bound structures are different to those with inhibitors [9], especially at the level of the recognition of peptide side chains in dedicated cavities (Figure 1A). These cavities are formed with the second GNAT fold, which is adapted to host and select the first six residues (Gly2 to aa6). Peptides with a Lys or Arg at position aa7 form a salt bridge with conserved Asp residues at the cavity entrance. The peptidesubstrate backbone adopts an extended structure and only promotes significant contacts with the enzyme at the outer side (aa7-9), explaining why octapeptides are optimal for NMT-driven 82 MYR.

83 Figure 1A shows the various NMT pockets. Pocket 1 specifically recognizes the long linear 84 hydrophobic chain of myristate. The first ten carbons are embedded within a linear pocket 85 formed from the side chains borne by the $\beta \mathrm{e}-\alpha \mathrm{C}-\beta \mathrm{f}$ adjacent secondary structures of the first 86 domain [10]. Each side chain's hydrophobic character is conserved, optimizing the pocket for 87 myristate recognition and excluding bulkier fatty acids (e.g., palmitate (C:16) or stearate (C:18)) 88 and branched fatty acids from isoprenoids such as farnesyl (C:15). The use of a linear fatty acid, 89 not synthesized in Archaea, might indicate that NMTs eventually acquired their lipid specificity 90 after engulfing bacterial partners that introduced the pathway (LACA; [11]).

91 Narrow pocket 2 is involved in $\mathrm{NH}_{2} \mathrm{Gly} 2$ recognition, pocket 3 favors small- and medium-sized 92 residues [7], and large pockets 4/5 can accommodate almost any chain. The narrow constriction 93 at the end of the peptide recognition pocket 6 favors small residues, especially Ser. While the 
94 emerging peptide recognition pattern (Figure 1) defines global specificity, it does not fully define

95 the actual substrate landscape in a given proteome.

96 Metabolic labeling

97 Chemical biology strategies coupled with mass spectrometry have been used to investigate 98 myristoylomes. The approaches involve, for instance, metabolic strategies that combine fatty acid analogs as precursors of myristoyl-CoA (MyrCoA) bearing a bio-orthogonal alkyne with NMT-

100 specific inhibitors [12] to identify NMT substrates in one shot in various organisms (Table 1, Key 101 Table). These approaches were subsequently completed with subcellular fractionation 102 approaches in plants. Combining the 364 sequences from these studies revealed a similar profile 103 (Figure 1B) to those observed in each of the nine organisms [7, 13-19].

104 Peptide arrays and artificial intelligence complete the myristoylomes

105 MYR co-translational activity can be mimicked in vitro with short peptides recapitulating 106 nascent chains combined with an NMT-catalyzed MYR assay [20-22]. Providing insights into NMT 107 specificity based on a reduced, non-random dataset, these approaches are biased and poorly 108 predictive [23]. Recent strategies have leveraged large arrays of peptides derived from the N109 termini of open reading frames starting with Gly from Arabidopsis thaliana and Homo sapiens 110 proteomes. These data have validated the quasi-entire set of positives in both organisms (Table 111 1) and provided crucial information on the negative set. About $20 \%$ of Gly-starting proteins were 112 MYRed. Human NMT1 and NMT2 and the functional NMT from $A$. thaliana displayed identical 113 substrate specificity. Nevertheless, Saccharomyces cerevisiae and Plasmodium falciparum NMTs 114 had restricted specificities in vitro and in vivo, partly assigned to negative residues when occurring 115 at aa8 and/or aa9 [22-24].

116 This sequence dataset was used to check NMT selectivity. The pattern (Figure 1C), although 117 similar to that obtained with global approaches, was more robust due to the higher number of 118 positive sequences and the $60 \%$ negative sequences. This allowed a better understanding of their 119 selectivity and why some proteins, despite displaying favorable residues (e.g., N3/S6), are not 120 substrates (Box 1$)$. 
122 Early studies on NMT catalysis [8] revealed an ordered mechanism with initial MyrCoA 123 binding. Although similar to all other GNATs, the NMT mechanism was distinct, with the peptide's

124 ammonium group making direct contact with the carboxy-terminal group to trap the proton and

125 allow the amino group to react. However, the widely-spaced reactive ammonium and thioester 126 bond of MyrCoA suggested that it must fully flip to approach and bind to it. Recent 3D structures 127 of HsNMT1 in complex with canonical substrates delivered sequential snapshots of the entire 128 catalytic mechanism spanning the enzymatic pathway from substrates, intermediates, and 129 products. In these models, the peptide substrate is differently positioned in pockets $2-4$, while 130 the ammonium creates a water-mediated bond with the base [7]. The reaction is thus easier to 131 achieve without major conformational changes around the alpha carbon of Gly2. These structures 132 reveal a conserved water channel connecting the deeply buried active site to the surface and the 133 key role of the Ab-loop, on which NMT catalysis relies (Figure 2).

\section{Myristoylomes, MYR targets, and evolutionary boundaries}

136 The five main NMT subtypes in eukaryotes

137 NMTs display an average of $50 \%$ identity, and phylogenetic clustering of NMTs recapitulate 138 the eukaryotic tree (Supplemental Figure 1), falling into plant, animal, 139 apicomplexa/diatom/ameba, and fungi/englenozoa/microsporidian classes. Unicellular 140 organisms and invertebrates have one NMT gene, while land plants and vertebrates have two 141 (NMT1, NMT2) [25]. The two land plant NMTs cannot be distinguished from each other and 142 probably correspond to early gene duplication without true speciation. Vertebrate NMT1/2 143 display very similar substrate specificity $[7,26]$. Interestingly, caspase cleavage of the $\mathrm{N}$-terminal 144 extension, previously thought to favor ribosome binding, results in the targeting of human NMT2 145 to membranes and NMT1 to the cytosol [27].

146 NMT-catalyzed MYR is essential for cell compartmentation

147 In silico analyses predicted 46 targets in the S. cerevisiae myristoylome, a dozen of which were 148 essential during stationary phase when inactivated (Table 1) [8, 28]. In A. thaliana, three essential 
149 targets were identified during development [24, 29, 30], and inhibition of Rpt2 MYR induced 150 phenotypes indicating decreased proteasome activity in yeast [31]. Another important MYRed

151 protein is the protein kinase Vps15, which is a PI3 kinase complex I subunit involved in macro152 autophagy in eukaryotes. All these targets shuttle between the cytosol and other compartments 153 including the nucleus (Rpt2/Sip2/Ptc2), the PM (Gpa1), and the endosome (Arf1/Arf2/Vps20).

154 The weak binding energy provided by a myristate moiety suggests that MYR cannot maintain 155 stable protein-membrane interactions by itself [32]. MYR can, however, target proteins to 156 endomembranes, and cytosolic partitioning was demonstrated to be correlated with NMT's 157 catalytic efficiency in A. thaliana [32]. Therefore, MYR of less efficient NMT substrates is partial 158 and varies between MYRed proteins [7]. While the hydrophobic myristate group is crucial for 159 membrane localization, its $\mathrm{N}$-terminus position plays a key role. For instance, C-terminal 160 prenylation does not compensate for the endocytic function of a non-MYRed ADP-ribosylation 161 factor (ARF) variant [33].

162 MYR's involvement in protein shuttling and reversible membrane binding is regulated through 163 various signals which allow the protein to associate or dissociate from the lipid bilayer [34]. The 164 switch may involve, for instance, (i) GTP hydrolysis inducing conformational changes in ARFs [35], 165 (ii) $\mathrm{Ca}^{2+}$ binding to EF-hand calcium-sensor proteins [36], (iii) phosphorylation of a polybasic motif 166 involved in electrostatic interactions with membrane phospholipids, or (iv) depalmitoylation of 167 vicinal cysteine residues. Such reversible myristoyl switches control MYR-dependent subcellular 168 protein trafficking, distributing MYRed proteins to different compartments. For instance, the 169 mammalian protein kinase A undergoes nuclear-cytoplasmic shuttling through significant 170 deamination of N3 in muscles due to electronegative charges caused by the new D3 [37].

171 The PM localization induced by cysteine residue PAL in the vicinity of the MYR site was initially 172 discovered for the mammalian homolog of Gpa1 [38, 39] and later in yeast [40]. This phenomenon 173 was recently confirmed by hierarchical clustering of protein accumulation profiles, which 174 revealed a clear shift in MYRed protein abundance toward PM fractions in the presence of second 175 targeting signals including PAL or polybasic tracks [19]. Yeast only features four such proteins 176 including Gpa2, and only Gpa1 is essential (Table 1). The proximal PAL of Gpa1 is therefore likely 
177 to be an ancient function linking MYR, PAL, and PM localization, probably later in evolution than 178 endosomes and relating to multi-cellularity and its required intercellular communication. Recent

179 studies on PAL and MYR co-occurrence in plants and humans have shown that PAL on cysteine 180 residues in the immediate vicinity of the MYRed Gly are readily detectable, with co-occurring 181 cysteine codons at positions 3-7 significantly more frequent in MYRed proteins. 50\% of MYRed 182 targets are expected to be palmitoylated (PALed) in plants and 23\% in humans [7].

\section{Perspectives with respect to eukaryogenesis}

184 The myristoylomes of nine organisms displaying NMTs of all classes (Table 1) allow us to draw 185 conclusions about the specific features promoted by NMTs throughout evolution. Figure 3A 186 shows that larger proteomes correspond to larger myristoylomes, which always constitute about $1872 \%$ of the proteome. The yeast myristoylome is an outlier, probably due to the gene loss 188 characterizing fungal evolution [3]. Nevertheless, Trypanosomatidae and Apicomplexa, which 189 also lost genes through parasitism [3], do not have similarly small myristoylomes, so the small 190 myristoylome observed in yeast might be partly linked to the restricted substrate specificity of 191 yeast NMT [24]. This is similar to yeast N-acetylation patterns, which are similarly subtly reduced, 192 especially as no N-acetylation Gly-starting proteins have been identified. Note that fungi still 193 display branch-specific essential MYR targets such as the GTPase EGO (Table 1) involved in micro194 autophagy.

195 Table 1 shows that a subset of eight yeast myristoylome proteins are also present throughout 196 all cell compartments of the myristoylomes of other organisms. The myristoylome has, therefore, 197 exploited all compartments elaborated by eukaryogenesis (Table 2). In addition, the major 198 eukaryotic sensing events also involve MYRed targets (Table 2). As a result, the myristoylome 199 illustrates the various steps of eukaryogenesis and explains why myristoylome size closely mirrors 200 that of the proteome (Figure 3A).

202 Proteome-wide approaches reveal unexpected targets

203 These high-throughput data have revealed that MYR targets unexpected compartments and 204 functions, especially catalytic processes, cell organization, and sensing (Figure 3B,C) but not gene 
205 expression and processes characterizing the first eukaryotic signature proteins (ESPs) [41, 42]. 206 Therefore, NMT probably appeared after these early ESP emerged in Archaea, likely in LACA. They 207 are much more relevant to the compartmentalization events underpinning eukaryogenesis. 208 Kinases, small GTPases, proteostasis-related phosphatases, hydrolases, transferases, and calcium 209 sensors feature often as targets (Figure 3B, C). These unexpected processes are eukaryote specific 210 and further illustrate the tight link between MYR and eukaryogenesis, as detailed below.

211 The immune system and MHC presentation

212 The mammalian immune system is equipped with virus detection machinery via virus213 synthesized MYRed peptides [43]. This was first shown for HIV-Nef N-terminal pentapeptides, 214 which add to the antigen repertoire presented by major histocompatibility complex (MHC) class

215 I molecules [43]. The crystal structure of the complex reveals a groove suitable for 4-9 residue 216 peptides $[44,45]$.

217 As MYRed peptides are derived from protein components associated with viral or bacterial 218 pathogenicity, this raises the possibility of exploiting MYR for vaccines [43]. The relationship 219 between MYR and immunity is now firmly established in plants and animals [46, 47]. When 220 impaired in humans, such as in rheumatoid arthritis, the lack of MYR of AMPK eventually triggers 221 inflammation due to failed lysosomal targeting [48].

222 Cell motility

223 Cilia

224 The cilium is a sensory organelle occurring in vertebrates and invertebrates, including 225 euglenozoa. Protein trafficking to cilia implies the presence of lipid microdomains that help 226 overcome the lipid diffusion barrier and relies on polarized trafficking of post-Golgi vesicles [49]. 227 Lipid modification of the cilial proteome is widespread and includes MYRed proteins [50]. Of note 228 is the MYRed cystin/nephrocystin. Cilial interactions are mediated by the UNC119 cargo, which 229 appears to be a major coordinator of MYRed protein trafficking, including a Src family kinase 230 (SFK) and nephrocystin [51]. Both are targeted into cilia via UNC119-mediated trafficking [52]. 231 The X-linked human retinitis pigmentosa gene XRP2 encodes a GTPase-activating protein involved 232 in Golgi-cilia trafficking, leading to UNC119 release [53]. XRP2 has MYR and PAL modification sites 
233 featuring Cys3 and Ser6, with deletion of the latter causing disease by inhibiting MYR and further

234 PM localization [54]. Finally, calflagin is a MYRed/PALed calcium-binding protein in kinetoplastids.

235 PAL on Cys3 is required for cilial sorting, while MYR alone is sufficient for membrane targeting $236[55]$

237 The glideosome of apicomplexa

238 The complex life cycles of human parasites like apicomplexa alternate between 239 asexual/sexual and parasitic/free stages in various hosts or organs. The parasitic stage involves 240 complex host cell interactions. Parasites elaborate dedicated secretory compartments such as 241 micronemes and rhoptries to promote the required motility. The gliding machinery allows 242 parasites to cross cell barriers to exit infected cells in a process analogous to but distinct from 243 that caused by cilia.

244 MYR is strongly involved in gliding motility $[56,57]$. In the malaria parasite $P$. falciparum, this 245 involves GAP45, which is MYRed [58]. Of the rhoptry components, ARO and the cGMP-dependent 246 protein kinase PKG are retrieved, while the ISP1 and ISP3 are major MYRed proteins [59] that link 247 the machinery to actin and myosin to induce traction [60]. Finally, gliding motility signaling 248 requires the same calcium-dependent protein kinases found in plants, which are almost 249 systematically MYRed and PALed [60].

250 Mitochondria

251 There are $>20$ MYRed proteins in the human mitochondrion, either induced by Met removal 252 or caspase cleavage. Several target the cytosolic side of the outer mitochondrial membrane 253 (OMM). These include NADH-cytochrome $b_{5}$ reductase, Sam50 (a sorting and assembly 254 machinery complex component), MOSC1/2 through co-translational MYR [61], and several post255 translationally MYRed proteins (see below). TOM40 is a MYRed subunit of the mitochondrial 256 import receptor subunit lying within the OMM but with an $\mathrm{N}$-terminus protruding at the outer 257 side $[62,63]$. It was unexpected that MYRed proteins could exist within the intermembrane space, 258 including the MIC19 and MIC25 components of the mitochondrial contact site complex (MICOS, 259 also found in yeast; Table 1), which is crucial for maintaining crista integrity and mitochondrial 260 function [64], and other proteins of the inner membrane space such as complex I subunits of the 
261 respiratory chain including B18/NDUFB7 and NDUF4 [7]. How they reach the outer membrane 262 without a mitochondrial targeting signal is unknown. A similar feature was noted in plants with

263 (i) the yeast homolog Mia40 (a mitochondrial intermembrane space assembly machinery 264 component) lying at the inner membrane next to MICOS and interacting with TOM at the OMM 265 [65], and (ii) two prohibins involved in crista morphogenesis [7, 19].

266 One hypothesis on how a MYRed protein might access the intermembrane space comes from 267 studies of yeast MIC19, which undergoes MYR in the cytosol. By interacting with Tom20 of the 268 TOM complex at the OMM, MIC19 would be imported into the inner mitochondrial membrane 269 (IMM) by so-called entropy pushing [66]. By contrast, delivery of the MYRed Tom40, which 270 features several transmembrane helices which insert into the OMM, involves Hsp90 [67].

271 Finally, it is interesting that ER-mitochondrial crosstalk involves both lipid exchange and $\mathrm{Ca}^{2+}$ 272 regulation, with the MYRed calcium sensor NCS1 homolog to the yeast form described in Table 1 273 involved [68]. The link between mitochondrial contact sites, MICOS, calcium signaling, and MYRed 274 protein import [65] needs further detailed examination, especially as these are some of the most 275 conserved MYRed targets (Table 1).

276 MYR and proteostasis

277 The plant myristoylome reveals about 50 proteins annotated as RING finger, F-box, or 278 ubiquitin domain proteins and E3 ubiquitin ligases [7, 19], some of which localize to the nucleus 279 [19]. Among Arabidopsis proteins, the SKIP2 subunit of the SCF(ASK-cullin-F-box) E3 ubiquitin 280 ligase complex was found to serve as a substrate receptor for E3 ligases. Interestingly, Toxoplasma 281 TgFBO1 is an inner membrane complex MYRed component with an F-box domain that might bind 282 SKIP1 [69]. In humans, ten such MYRed E3 cullins were identified [7, 70]. The identification of 283 major MYRed components of protein degradation reveals MYR as an important feature of 284 proteostasis (see also glossary and below).

285 Apoptosis

286 Induction of apoptosis leads to the activation of numerous caspase proteases. In some cases, 287 caspase cleavage may expose internal cryptic MYR sites to the action of NMTs. MYR-dependent 288 relocalization to the OMM of fragments of BID and actin $[71,72]$ has been reported. BID switching 
contributes to enhanced cytochrome $c$ release and cell death [71]. A Huntingtin fragment also relocalized to the autophagosome due to MYR [73], while the MYRed gelsolin fragment is

291 cytosolic [74], and the MYRed C-terminal fragments of PAK2 and PKC $\varepsilon$ are redirected to internal 292 membranes and that of filensin to the PM [75-77]. A global approach has identified another 40 293 proteins MYRed following caspase cleavage. The MYR consensus is oversimplified while the caspase consensus strongly emerges (Figure 1D), suggesting that post-translational MYR was acquired later in the course of eukaryotic evolution and has not yet converged.

Ferroptosis suppressor protein 1 (FSP1/AIFM2) is a MYRed protein that localizes to the PM and behaves as an oxidoreductase, converting ubiquinone to ubiquinol to inhibit ferroptosis [78, 79]. This programmed cell death mechanism is conserved in plants [80] and leads to lipid damage and membrane degradation. Glutathione peroxidase GPX4 in vertebrates together with glutathione prevent lipid peroxidation. While the MYRed FSP1/AIFM2 acts as a parallel system to GPX4, of note, two of the eight GPX isoforms in plants (GPX4/5) are MYRed [19] so might act similarly to FSP1/AIFM2 directly at the PM. Also, plants have a large array of thioredoxins and

303 glutaredoxins featuring MYR [32].

\section{MYR and quality control}

A MYR, Gly-specific N-degron

Recent data show that two cullin-RING E3 ligases (CRL2 and CRL5) target Gly-starting peptides 308 for degradation [81]. Globally, Gly substrates favored by CRL2 included those starting with a Gly 309 followed by Phe, Gly, His, Leu, Met, or Tyr, while those followed by Asp, Glu, Ile, Asn, Pro, Arg, 310 Ser, or Thr were disfavored. Interestingly, metazoan proteomes seem to be depleted of CRL2311 mediated N-terminal Gly degrons, while CRL2 complexes most likely target protein fragments 312 bearing N-terminal Gly degrons after caspase cleavage during apoptosis. 13\% and 17\% of known 313 post-translationally MYRed proteins display a residue 3 favored and disfavored by CRL2, 314 respectively. Loss of MYR destabilized Gly-starting polypeptides followed by Cys, Gln, or Lys, so 315 the authors proposed that N-terminal Gly degrons might play a role in MYR quality control, 316 allowing degradation of proteins failing to undergo MYR and avoiding accumulation of non- 
317 MYRed "aberrant" species. However, partial stabilization of endogenous N-MYRed proteins after 318 loss of MYR including CRL2 adaptors suggests a requirement for additional E3 ligases and/or other

319 undetermined factors. Finally, it remains to be fully understood which non-MYRed Gly-starting 320 proteins are related to Gly degrons, as there clearly remains a non-negligible number of such

321 species that eventually accumulate in multicellular organisms as either $\mathrm{N} \alpha$-acetylated or with an 322 unmodified Gly [5, 7]. The favored CRL2 sequences indeed deal with $<14 \%$ of the human 323 myristoylome, while the disfavored sequences involve 51\% (see SupDataset 2 in [7]).

\section{Competition with other modifications or processes}

325 There is increasing evidence that one unique proteoform may undergo various modifications 326 at the same site within the same cell, including for MYRed proteins, which may be either partially 327 modified or partially N-acetylated [7]. MYR's pronounced effect on membrane binding is likely to 328 have a major effect on protein localization, as observed for mammalian NADH-cytochrome $b_{5}$ 329 reductase, which is targeted to the ER if $\mathrm{N \alpha}$ acetylated and the mitochondrion when MYRed [61]. 330 Roles of sirtuins and HDACs with Lys-MYR

$331 \quad$ NMTs are thought to exclusively acylate N-terminal Gly residues. Nevertheless, the $\varepsilon$-amino 332 groups of internal lysines of several proteins including tumor necrosis factor- $\alpha$, interleukin- $\alpha 1$ 333 precursor, and serine hydroxymethyltransferase 2 also undergo MYR [82, 83], although the 334 corresponding catalyst(s) still remain unidentified.

335 Interestingly, it was recently demonstrated that human NMTs may transfer myristate onto 336 the N-terminal epsilon amino groups of lysine side chains, expanding its known substrate range 337 beyond the well-established N-terminal alpha moiety of Gly peptides [84]. Furthermore, Lys3 338 MYR allows ARF6 to remain on membranes during GTPase cycling [85]. In contrast to the 339 irreversible Gly-MYR, several acyl hydrolases targeting Lys-MYR have recently been described 340 including Sirtuin6, HDAC8, and HDAC11 [82], making Lys-MYR a reversible modification 341 dependent on these specific hydrolases. This implies that Lys-MYR, as with other reversible 342 modifications (i.e., phosphorylation, $\mathbf{N}$-terminal acetylation), might be intimately related to 343 signal transduction and tightly regulated by as yet unknown biotic or abiotic signals. In the case 344 of ARF6, NMT prefers the GTP-bound form of ARF6 while SIRT2 prefers the GDP-bound form, 
345 allowing an NMT/SIRT2-ARF6 regulatory axis of the GTPase cycle [85]. The N-terminal Lys-MYR 346 might be part of an N-degron, so an important role of Lys-MYR deacylases could be to ensure 347 protein quality control by preventing intracellular Lys-MYR accumulation [84].

\section{Concluding remarks}

350 Four decades of seminal contributions have now contributed to knowledge about the key 351 MYR process, with recent studies on MYR and NMT biology leading to revisiting and rethinking 352 old dogmas. Multiscale approaches have proven pivotal in expanding our knowledge of

353 myristoylomes. Recent efforts to precisely annotate all proteoforms in several proteomes have 354 been valuable for comprehensive MYR mapping [7]. Future studies will increase the actual 355 myristoylome with all possible substrates resulting from post-translational MYR. This new pool of 356 post-translational MYRed substrates can be unraveled taking into account protein substrate 357 specificity ensured by the newly identified specific NMT pockets, the amino acid pattern of the 358 identified MYRed proteins, and protease cleavage specificity. This will reveal whether the pools 359 of co- and post-translational Gly-Myr share the same N-terminal features.

360 Gly-myristoylome characterization has also revealed that a non-negligible number of MYRed 361 substrates are partially modified by MYR and that the same N-terminal Gly can undergo different 362 modifications, completely altering the protein's properties. The factors influencing specific 363 modifications and the impact of quality control involving Gly N-degrons and E3 ligases are still 364 unknown, but future elucidation will prove crucial for understanding both the involved factors 365 and their biological roles.

366 MYR was long considered an irreversible modification. However, myristoyl-glycine cleavage 367 by Shigella protease IpaJ was recently described $[86,87]$, opening up new directions on the 368 dynamic regulation of MYR. The plant hormone ABA was proposed to inhibit MYR, in turn 369 triggering relocalization of several protein targets [88]. In this context, we anticipate that NMT 370 activity may be insufficient to modify all protein substrates and MyrCoA donor availability may 371 also be insufficient, while dysregulation of de novo synthesis might accelerate the growth of 372 tumors that express high levels of activated Src kinase [89]. 
373 Finally, the recent discovery of a novel NMT catalytic mechanism suggesting MYR of N374 terminal Lys in addition to Gly takes the field into an exciting era where mechanisms of MYR

375 regulation, biological effects, and pathological consequences become central aspects.

377 Acknowledgements

378 The team was funded by Agence Nationale de la Recherche (ANR-2010-BLAN-1611-01) and 379 Fondation ARC (SFI2011120111203841) and benefitted from the support of the Labex Saclay Plant 380 Sciences-SPS (ANR-10-LABX-0040-SPS).

382 Glossary

$38326 \mathrm{~S}$ proteasome: a $2.5 \mathrm{MDa}$ molecular machine controlling ubiquitin-dependent proteolysis in 384 eukaryotes made of two (19S-regulatory and 20S-core proteolytic) complexes.

385 ADP-ribosylation factors (ARFs):, small GTPases essential in eukaryotes that function in vesicular 386 trafficking and actin remodeling.

387 Autophagy: mechanism allowing degradation or recycling of cellular components by fusion to 388 lysosomes.

389 Co-translational: an event occurring while the nascent protein chain is not yet complete and still 390 bound to the ribosome

391 Degron: specific sequence in a protein that directs its degradation via the 26S proteasome or 392 autophagy.

393 Endosomal sorting complex required for transport (ESCRT):, a set of three complexes. ESCRT-III 394 consists of 8-12 subunits including Vps20/CHMP6.

395 ESP: eukaryotic signature proteins, early markers of eukaryogenesis and including DNA, RNA, and 396 protein synthesis proteins but also ESCRT, small GTPases, cytoskeletal proteins, and ubiquitin 397 signaling proteins.

398 Eukaryogenesis: approximately 2-billion-year process by which eukaryotes evolved from an 399 Archaeon. LACA and FECA witnessed progressive emergence of endomembranes, nuclei, and 400 organelles. 
401 Evolution: all life on Earth has evolved from common ancestors in an unbroken chain since its 402 origin, approximately 3.8 billion years ago.

403 FECA: first eukaryotic common ancestor, a protoeukaryote with endomembranes (approx. 2.4 404 billion years ago).

405 GNATs: GCN5-related N-acetyltransferases, a large enzyme superfamily that transfer acyl 406 derivatives from acyl-CoA donors to a variety of acceptors.

407 GTPases: hydrolyze GTP into GDP, include small proteins like ARFs or large ones such as 408 heterotrimeric $\mathrm{G}$ proteins, major molecular switches of signal transduction pathways located at 409 the PM.

410 LACA: last archaeal common ancestor (approx. 2.8 billion years ago), an archaeon featuring ESPs

411 such as cytoskeletal proteins and membrane trafficking systems.

412 LECA: last eukaryotic common ancestor, featuring fully differentiated internal structures (approx. 4131 billion years ago).

414 Methionine aminopeptidases: enzymes cleaving the first methionine of approx. $60 \%$ proteins to 415 unmask small residues such as glycines.

416 Major histocompatibility complex: cell surface proteins essential for vertebrate acquired 417 immunity.

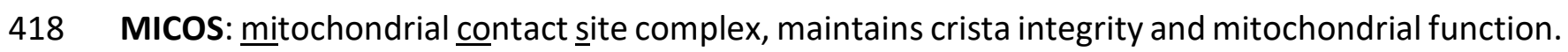

419 MYR: N- $\alpha$-myristoylation, a lipid acylation of proteins using MyrCoA as a myristate donor; makes 420 amide bonds with free amino groups, usually from $\mathrm{N}$-terminal glycines.

421 MYRed: N-myristoylated, refers to glycine MYR in large-scale analyses.

422 Myristate, a saturated (i.e., without double bonds) 14 carbon fatty acid (C14:0).

423 Myristoylome: complete set of MYRed proteins in a proteome.

424 NAA: enzymes of the GNAT family, which use acetyl CoA as a donor to acetylate the alpha amino425 termini of proteins. The catalytic subunit may be associated with auxiliary subunits to form $\mathrm{N}-\alpha-$ 426 acetyltransferase complexes (NATs). 
427 Naa10: catalytic subunit of $\mathrm{N}$ - $\alpha$-acetyltransferase $\mathrm{A}$ (NatA), an NAA operating on small N-terminal 428 residues such as glycine.

429 NMT: N-myristoyltransferase, the MYR catalyst, part of the GNAT family, uses MyrCoA as donor. $430 \mathrm{~N}$-terminal acetylation: a modification occurring on the $\mathrm{N}$-termini of proteins. Various complexes 431 have been described, such as NatA and NatB, depending their substrate specificity. Involves $80 \%$ 432 of proteins of multicellular eukaryotes.

433 PAL: S-palmitoylation next to MYR; catalyzed by membrane enzymes, occurs anywhere on 434 cysteine residues provided a membrane-binding motif (transmembrane helix/helices, lipid 435 modification) brings the residue to the interface.

436 Proteoform: protein derivative from one gene, may differ by one modification.

437 Proteostasis: the proteome steady-state homeostasis as guaranteed by a network of quality 438 control components ensuring proteins integrity.

439 SFK: Src family kinase, non-receptor Src family of protein tyrosine kinases, nine members, none 440 in plants. 
Text Boxes

\section{Box 1: Protein modification probability and frequency}

443 Common representations of the substrate selectivity of enzymes involved in protein

444 modifications such as proteases use extremely useful logos and powerful visualizations that 445 integrate massive data into a single, striking image [90]. Nevertheless, care must be taken when 446 drawing conclusions given the complexity associated with the recognized pattern, which takes

447 into account: (i) various positive and negative effects of the enzyme; (ii) evolutionary constraints 448 from which the initial non-MYRed target starts; (iii) functional and structural constraints of the N449 terminal domain, which may be required for in vivo activity (interaction, folding, catalysis, other 450 modifications); and (iv) the fact that there are other constraints from the genetic message and its 451 interpretation by a specific organism (codon usage, amino acid availability, RNA folding, 452 completion with other enzymes including methionine aminopeptidases and NAAs). Figure I 453 exemplifies this feature. From Figure 1, one can easily identify the main features of the NMT 454 recognition site (for instance $\mathrm{C}, \mathrm{N}$, or $\mathrm{Q}$ at position 3 ). If one compares the positives from global 455 analysis in blue and macroarray results in green in Figure I, the overall frequency is clearly 456 identical. Still, considering the most obvious element of selectivity at position 6 (with $\mathrm{S}$ being a 457 feature) (Figures 1 \& 2), they only represent $60 \%$ of the complete set. With the help of the 458 negative microarray set, one can analyze the probability that an extracted genomic sequence is 459 MYRed (orange). The data show that there is no single amino acid which leads to a frequency 460 higher than $82 \%$ with N3 (Figure I). Still, such proteins only represent $25 \%$ of the entire set. 461 Combination increases the probability to $93 \%$ (N3/S6), but those proteins are only $5 \%$ of all 462 positives.

463 The difficulty in drawing decision trees from amino acid patterns explains why artificial 464 intelligence approaches including machine learning strategies are required for prediction [7]. 465 Such tools need not only positive but negative sets to ideally delineate, learn, and distinguish a 466 positive from a negative. It is important to remember that the theoretical diversity with 7 amino 467 acids is huge $\left(10^{9}\right)$, and current approaches still deal only with hundreds of data points or two 468 thousand in the case of the macroarray. Obtaining negative sets with MS approaches based on 
469 positive selection is only feasible if combined strategies involving diverse selection procedures

470 are used. A strategy involving the identification of proteins with non-modified or N $\alpha$-acetylated

$471 \mathrm{~N}$-termini was used to obtain information on proteins starting with non-MYRed glycines in vivo $472[7]$.

473 Legend to Figure I in Box 1: relationships between the probability and frequency of MYR

474 Data are derived from the peptide array [7] of 2,066 sequences including 837 positives and all 475 data from click chemistry (365 sequences). \% positives click refers to the click chemistry set. \% 476 positives macroarray (green bars) refers to the frequency of positives in the positive set of 837 477 sequences. Probability of being positive or true positives (orange bars) refers to the frequency of 478 positives in the complete array of 2,066, defining the probability of being MYRed. 


\section{Figure Legends}

482 Figure 1. Specific pockets of NMTs ensure protein substrate specificity

483 Panel A. Various structures of human NMT1 crystallized with MyrCoA and one of 5 different 484 peptide substrates (PDB IDs: 509S, 509V, 509U, 6EHJ, 6QRM) superimposed to show how the 485 various side chains fill each pocket. Each pocket is delineated with a full line and labeled (see text). 486 Panel B. Corresponds to 365 octapeptides extracted from N-terminal sequences from MYRed 487 proteins identified by global approaches with a negative set against Swiss-Prot (Homo sapiens 488 was used as the proteome as very similar results were obtained with other proteomes).

489 Panel C A data set of a macroarray made of $>2,000$ octapeptides assayed in vitro with NMT is 490 available in [7]. The panel displays the positive data (836) against the negatives $(1,229)$. Gly2 does 491 not appear as it represents $>99 \%$ of the entire dataset, but " $G$ " was added for the sake of easier 492 comparison with panel A.

493 Panel D. Post-translational MYR recognition pattern. The panel displays residues around the 494 modified glycine (residue 5 before cleavage and MYR). The data are compiled from SupData4 in 495 [13]. The sequence of the filensin C-domain was added to build the pattern. Panels B-D are 496 independent and were built with iceLogo 1.2 [90].

Figure 2: Pre-organized water molecules in a dedicated conserved channel contribute to the catalytic mechanism

501 Top, the NMT water channel is schematized. Flux directions of water and protons are indicated in 502 blue and red, respectively. The two proton donors are indicated on the right side. Water 503 molecules are numbered from the outer to the inner side of the active site (W12 to W1).

504 Center, superimposition of the water molecules of the channel and active site in HsNMT1:MyrCoA 505 and HsNMT1:MyrCoA:peptide states. The organized water molecules in the peptide-free state 506 shown in purple and those in the peptide-binding, tetrahedral intermediate state in red. The 
507 volume of the water channel is drawn in grey. The MyrCoA is shown as ball and sticks and colored

508 in orange. Hydrogen bonds are shown as dashed lines.

509 Middle box, left, organization of the exit of the water channel with three residues contributing to

510 a hydrogen bond network including the conserved Y356; right, close up of the active site in an

511 alternative view. W1-3 are displayed at the two states to show how a new water molecule (W3)

512 is pushed into the active site while entering through the water channel at TI.

513 Bottom box, right. Close-up of the active site around T282 and Q496. Detailed hydrogen bond

514 network around W1-W5.

515 Bottom box, left. Operating principle of a proton wire semiconductor: the protons hop along

516 chains of hydrogen bonds between water molecules making intermediary hydronium ions

517 (adapted from [91]). Four steps of proton transfers figured in this cartoon of a wire made of 5

518 water molecules. A wire can be made of two and more than ten water molecules.

519

520 Figure 3: Myristoylome sizes and the categories of MYRed proteins in multicellular organisms

521 Panel A. Linear relationship between proteome and myristoylome sizes. The data from Table 1

522 were plotted. The dot in grey corresponds to the yeast data, which is an outlier featuring a 523 reduced myristoylome (see text).

524 Panel B \& C. Categories of MYRed proteins in multicellular organisms. Only those proteins for 525 which gene ontology information is available were analyzed. 439 proteins sorted from $A$. thaliana 526 (Panel B). 221 proteins sorted from H. sapiens (Panel C). 
528 Tables

529 Table 1, Key Table: Myristoylome sizes and conservation of major MYR targets throughout

530 species

531 Proteomes were extracted from Uniprot (https://www.uniprot.org/); the Gly-ome corresponds

532 to all ORFs displaying a Gly2 residue. Green boxes refer to conserved targets throughout species.

$533{ }^{f}$ data from GT1 strain consolidated and confirmed with data from T. gondii strains ME49 and

534 FOU. All data are from ToxoDB 36 release.

535 \$library likely cumulating all proteoforms.

$536 \quad$ f value calculated from data obtained with the machine learning predictor described in [7]. The

537 values can be compared to those published with another predictor dating to 2002 with far less

538 robust genomic annotation (see Table 6 in Ref.[92]). The performances of various predictors

539 available online are compared in Table 1 of Ref.[23].

540 \& data from individual or large-scale analysis (with exception of $T$. gondii, see [93]); data in

541 parentheses refer to those obtained with peptide libraries derived from genuine protein

542 sequences, see references [7, 13-19]. For $A$. thaliana and $H$. sapiens, see Supplementary Datasets

$5431 \& 2$ in Ref.[7].

544 \# data taking into account all validated proteoforms from NextProt2017

545 (https://www.nextprot.org/) and AraProt2017 (https://www.arabidopsis.org/index.jsp).

$546{ }^{*}$ means that an ORF with strong homology, Gly2, and a likely MYR consensus is retrieved in the 547 proteome.

$548{ }^{* *}$ the protein exists but not with a Gly2; homologs of the apicomplexan phylum (Babesia spp.)

549 display a Gly2 followed by a good MYR consensus. 


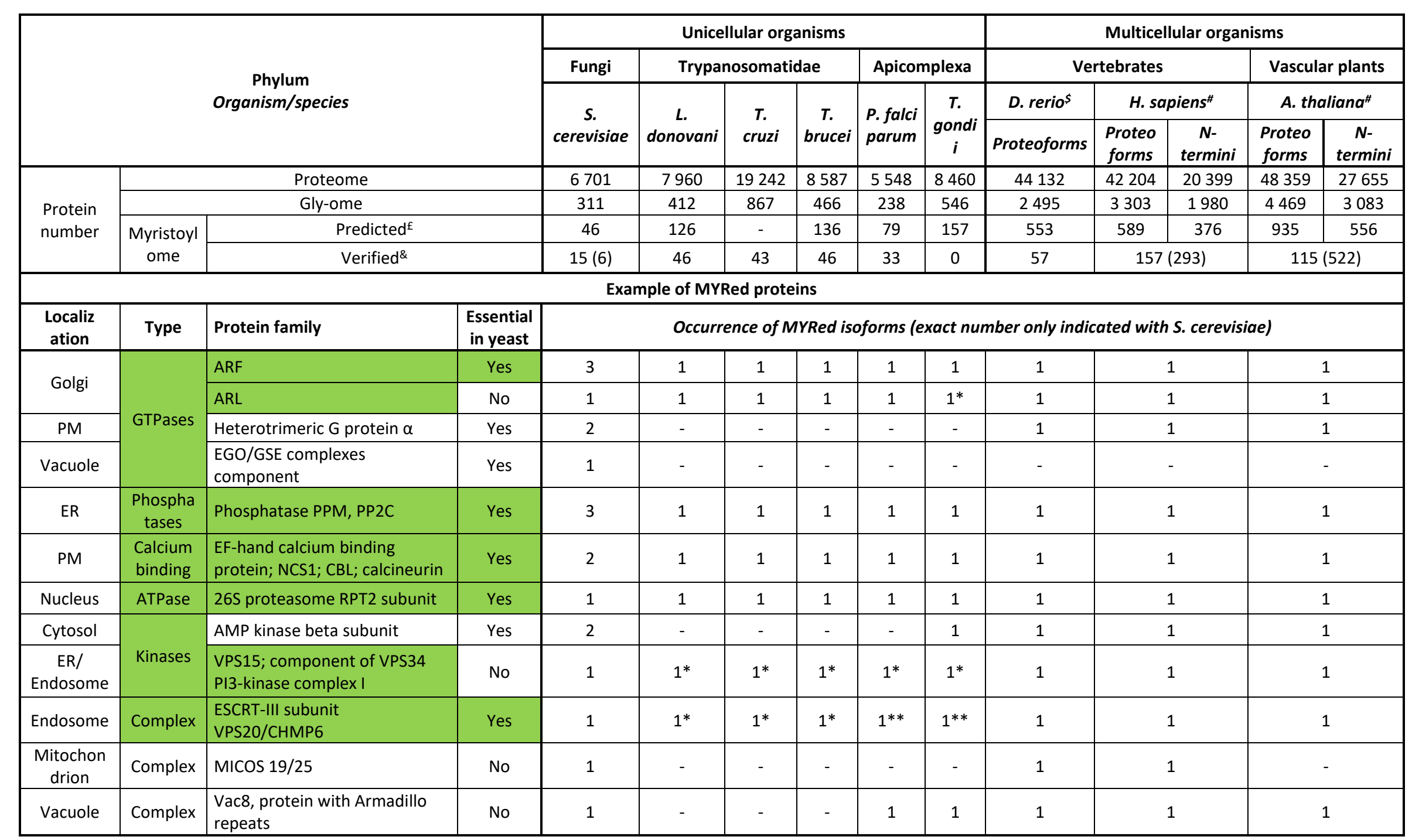


551 Table 2: NMT, its targets, and the major events in eukaryotic evolution

\begin{tabular}{|l|l|}
\hline Stage of eukaryogenesis & MYR event \\
\hline Protoeukaryote & $\begin{array}{l}\text { Early NMT evolution from a prokaryotic } \\
\text { GNAT acetyltransferase }\end{array}$ \\
\hline Emergence of endomembranes (ER, Golgi) & ARFs act as early NMT targets \\
\hline Early cell division & ESCRTIII Vps20/CHMP6 involved \\
\hline Creation of nuclear endomembrane & $\begin{array}{l}\text { 26S proteasome regulatory subunits are } \\
\text { shuttled together with Rpt2 }\end{array}$ \\
\hline $\begin{array}{l}\text { Aerobic bacterium engulfment, allowing oxygen } \\
\text { accommodation and energy sensing }\end{array}$ & $\begin{array}{l}\text { AMK/Sip1/SnRK kinase assemble as } \\
\text { heterotrimeric complexes }\end{array}$ \\
\hline Calcium as major signal transducer & Calcium sensing with calcineurin-like proteins \\
\hline $\begin{array}{l}\text { ER stress, regulation of the unfolded protein } \\
\text { response }\end{array}$ & PP2C phosphatases involved \\
\hline Vacuole sorting, macroautophagy & Protein kinase Vps15 \\
\hline $\begin{array}{l}\text { Mitochondria become symbiotic organelles, gene } \\
\text { transfer to the nucleus }\end{array}$ & $\begin{array}{l}\text { MICOS complex ensures link between ER and } \\
\text { mitochondria }\end{array}$ \\
\hline $\begin{array}{l}\text { Early cell differentiation allows cell communication } \\
\text { through membrane receptors and allow sex mating } \\
\text { in fungi, for instance; leads to multicellularity }\end{array}$ & $\begin{array}{l}\text { Heterotrimeric G alpha subunit, interaction } \\
\text { with PI3 kinase/phosphodiesterase activation }\end{array}$ \\
\hline Animal cell differentiation & Tyrosine kinases of the SFK family appear \\
\hline $\begin{array}{l}\text { Plastids become symbiotic organelles } \\
\text { formate }\end{array}$ \\
\hline
\end{tabular}




\section{References}

554 1. Meinnel, T. and Giglione, C. (2008) Protein lipidation meets proteomics. Front Biosci 13, 6326-

55540.

556 2. Resh, M.D. (2016) Fatty acylation of proteins: The long and the short of it. Prog Lipid Res 63,

$557 \quad 120-31$

558 3. Dacks, J.B. and Field, M.C. (2018) Evolutionary origins and specialisation of membrane 559 transport. Curr Opin Cell Biol 53, 70-76.

560 4. Aksnes, $\mathrm{H}$. et al. (2019) Co-translational, post-translational, and non-catalytic roles of N-

561 terminal acetyltransferases. Mol Cell 73 (6), 1097-1114.

562 5. Arnesen, T. et al. (2009) Proteomics analyses reveal the evolutionary conservation and

563 divergence of N-terminal acetyltransferases from yeast and humans. Proc Natl Acad Sci U S A 106

564 (20), 8157-62.

565 6. Bienvenut, W.V. et al. (2012) Comparative large scale characterization of plant versus mammal

566 proteins reveals similar and idiosyncratic N-alpha-acetylation features. Mol Cell Proteomics 11

567 (6), M111 015131.

568 7. Castrec, B. et al. (2018) Structural and genomic decoding of human and plant myristoylomes

569 reveals a definitive recognition pattern. Nat Chem Biol 14 (7), 671-679.

570 8. Bhatnagar, R.S. et al. (2001) Biology and enzymology of protein N-myristoylation. In The

571 enzymes (Tamanoi, F. and Sigman, D.S. eds), pp. 241-286, Academic Press. 
572 9. Farazi, T.A. et al. (2001) Structures of Saccharomyces cerevisiae N-myristoyltransferase with

573 bound myristoylCoA and peptide provide insights about substrate recognition and catalysis.

574 Biochemistry 40 (21), 6335-43.

575 10. Bhatnagar, R.S. et al. (1999) The structure of myristoyl-CoA:protein N-myristoyltransferase.

576 Biochim Biophys Acta 1441 (2-3), 162-72.

577 11. Koonin, E.V. (2015) Origin of eukaryotes from within archaea, archaeal eukaryome and bursts

578 of gene gain: eukaryogenesis just made easier? Philos Trans R Soc Lond B Biol Sci 370 (1678),

57920140333.

580 12. Lanyon-Hogg, T. et al. (2017) Dynamic protein acylation: new substrates, mechanisms, and 581 drug targets. Trends Biochem Sci 42 (7), 566-581.

582 13. Thinon, E. et al. (2014) Global profiling of co- and post-translationally N-myristoylated 583 proteomes in human cells. Nat Commun 5, 4919.

584 14. Wright, M.H. et al. (2014) Validation of N-myristoyltransferase as an antimalarial drug target 585 using an integrated chemical biology approach. Nat Chem 6 (2), 112-21.

586 15. Broncel, M. et al. (2015) Multifunctional reagents for quantitative proteome-wide analysis of 587 protein modification in human cells and dynamic profiling of protein lipidation during vertebrate 588 development. Angew Chem Int Ed Engl 54 (20), 5948-51. 
589 16. Wright, M.H. et al. (2015) Global analysis of protein N-myristoylation and exploration of N590 myristoyltransferase as a drug target in the neglected human pathogen Leishmania donovani.

591 Chem Biol 22 (3), 342-54.

592 17. Wright, M.H. et al. (2016) Global profiling and inhibition of protein lipidation in vector and 593 host stages of the sleeping sickness parasite Trypanosoma brucei. ACS Infect Dis 2 (6), 427-441.

594 18. Roberts, A.J. and Fairlamb, A.H. (2016) The N-myristoylome of Trypanosoma cruzi. Sci Rep 6, 59531078.

596 19. Majeran, W. et al. (2018) Targeted profiling of A. thaliana sub-proteomes illuminates new co597 and post-translationally N-terminal Myristoylated proteins. Plant Cell 30 (3), 543-62.

598 20. Towler, D.A. et al. (1988) The biology and enzymology of eukaryotic protein acylation. Annu. 599 Rev. Biochem. 57, 69-99.

600 21. Boutin, J.A. (1997) Myristoylation. Cell Signal 9 (1), 15-35.

601 22. Boisson, B. et al. (2003) Unexpected protein families including cell defense components 602 feature in the N-myristoylome of a higher eukaryote. J Biol Chem 278 (44), 43418-43429.

603 23. Traverso, J.A. et al. (2013) High-throughput profiling of N-myristoylation substrate specificity 604 across species including pathogens. Proteomics 13 (1), 25-36.

605 24. Pierre, M. et al. (2007) N-Myristoylation regulates the SnRK1 pathway in Arabidopsis. Plant 606 Cell $19(9), 2804-21$. 
607 25. Giang, D.K. and Cravatt, B.F. (1998) A second mammalian N-myristoyltransferase. J. Biol. 608 Chem. 273 (12), 6595-6598.

609 26. Rundle, D.R. et al. (2002) Characterization of type I and type II myristoyl-CoA:protein N610 myristoyltransferases with the Acyl-CoAs found on heterogeneously acylated retinal proteins. Exp 611 Eye Res 75 (1), 87-97.

612 27. Perinpanayagam, M.A. et al. (2013) Regulation of co- and post-translational myristoylation of 613 proteins during apoptosis: interplay of N-myristoyltransferases and caspases. FASEB J 27 (2), 811 61421.

615 28. Ashrafi, K. et al. (1998) A role for Saccharomyces cerevisiae fatty acid activation protein E in 616 regulating $N$-myristoylation during entry into stationary phase. J Biol Chem 273 (40), 2586461725874.

618 29. Bayer, M. et al. (2009) Paternal control of embryonic patterning in Arabidopsis thaliana. 619 Science 323 (5920), 1485-8.

620 30. Renna, L. et al. (2013) Golgi traffic and integrity depend on N-myristoyl transferase-1 in 621 Arabidopsis. Plant Cell 25 (5), 1756-73.

622 31. Kimura, A. et al. (2012) N-myristoylation of the Rpt2 subunit regulates intracellular localization 623 of the yeast $26 \mathrm{~S}$ proteasome. Biochemistry 51 (44), 8856-66. 
624 32. Traverso, J.A. et al. (2013) Roles of N-Terminal fatty acid acylations in membrane 625 compartment partitioning: Arabidopsis h-type thioredoxins as a case study. Plant Cell 25 (3), $626 \quad 1056-77$.

627 33. D'Souza-Schorey, C. and Stahl, P.D. (1995) Myristoylation is required for the intracellular 628 localization and endocytic function of ARF6. Exp Cell Res 221 (1), 153-9.

629 34. Resh, M.D. (2006) Trafficking and signaling by fatty-acylated and prenylated proteins. Nat 630 Chem Biol 2 (11), 584-90.

631 35. Goldberg, J. (1998) Structural basis for activation of ARF GTPase: mechanism of guanine 632 nucleotide exchange and GTP-myristoyl switching. Cell 95, 237-248.

633 36. Ames, J.B. and Lim, S. (2012) Molecular structure and target recognition of neuronal calcium 634 sensor proteins. Biochim Biophys Acta 1820 (8), 1205-13.

635 37. Pepperkok, R. et al. (2000) Intracellular distribution of mammalian protein kinase A catalytic 636 subunit altered by conserved Asn2 deamidation. J Cell Biol 148 (4), 715-26.

637 38. Parenti, M. et al. (1993) A novel N-terminal motif for palmitoylation of G-protein $\alpha$ subunits. 638 Biochem J 291 (2), 349-353.

639 39. Degtyarev, M.Y. et al. (1993) The G protein .alpha.s subunit incorporates [3H]palmitic acid and 640 mutation of cysteine-3 prevents this modification. Biochemistry 32 (32), 8057-8061. 
641 40. Song, J. and Dohlman, H.G. (1996) Partial constitutive activation of pheromone responses by

642 a palmitoylation-site mutant of a G protein alpha subunit in yeast. Biochemistry 35 (47), 14806-

64317.

644 41. Koumandou, V.L. et al. (2013) Molecular paleontology and complexity in the last eukaryotic 645 common ancestor. Crit Rev Biochem Mol Biol 48 (4), 373-396.

646 42. Spang, A. et al. (2015) Complex archaea that bridge the gap between prokaryotes and 647 eukaryotes. Nature 521 (7551), 173-179.

648 43. Morita, D. and Sugita, M. (2016) Lipopeptides: a novel antigen repertoire presented by major 649 histocompatibility complex class I molecules. Immunology 149 (2), 139-145.

650 44. Morita, D. et al. (2016) Crystal structure of the N-myristoylated lipopeptide-bound MHC class 651 I complex. Nat Commun 7, 10356.

652 45. Yamamoto, Y. et al. (2019) Identification and structure of an MHC class I-encoded protein 653 with the potential to present N-myristoylated 4-mer peptides to T cells. J Immunol 202 (12), 33496543358.

655 46. Rowe, D.C. et al. (2006) The myristoylation of TRIF-related adaptor molecule is essential for 656 Toll-like receptor 4 signal transduction. Proc Natl Acad Sci U S A 103 (16), 6299-6304.

657 47. Udenwobele, D.I. et al. (2017) Myristoylation: an important protein modification in the 658 immune response. Front Immunol 8, 751. 
659 48. Wen, Z. et al. (2019) N-myristoyltransferase deficiency impairs activation of kinase AMPK and 660 promotes synovial tissue inflammation. Nat Immunol 20, 313-325.

661 49. Nachury, M.V. et al. (2010) Trafficking to the ciliary membrane: how to get across the 662 periciliary diffusion barrier? Ann Rev Cell Dev Biol 26 (1), 59-87.

663 50. Roy, K. and Marin, E.P. (2019) Lipid modifications in cilia biology. J Clin Med 8 (7), 921.

664 51. Constantine, R. et al. (2012) Uncoordinated (UNC)119: coordinating the trafficking of 665 myristoylated proteins. Vision Res 75, 26-32.

666 52. Stephen, L.A. et al. (2018) The ciliary machinery is repurposed for T cell immune synapse 667 trafficking of LCK. Dev Cell 47 (1), 122-132.e4.

668 53. Wright, K.J. et al. (2011) An ARL3-UNC119-RP2 GTPase cycle targets myristoylated NPHP3 to 669 the primary cilium. Genes Dev 25 (22), 2347-2360.

670 54. Chapple, J.P. et al. (2000) Mutations in the N-terminus of the X-linked retinitis pigmentosa 671 protein RP2 interfere with the normal targeting of the protein to the plasma membrane. Hum 672 Mol Genet $9(13), 1919-1926$.

673 55. Emmer, B.T. et al. (2009) Identification of a palmitoyl acyltransferase required for protein 674 sorting to the flagellar membrane. J Cell Sci 122 (6), 867-874.

675 56. Doerig, C. et al. (2015) Post-translational protein modifications in malaria parasites. Nat Rev 676 Microbiol 13 (3), 160-72. 
677 57. Schlott, A.C. et al. (2018) N-Myristoylation as a drug target in malaria: exploring the role of N678 myristoyltransferase substrates in the inhibitor mode of action. ACS Infect Dis 4 (4), 449-457.

679 58. Rees-Channer, R.R. et al. (2006) Dual acylation of the $45 \mathrm{kDa}$ gliding-associated protein 680 (GAP45) in Plasmodium falciparum merozoites. Mol Biochem Parasitol 149 (1), 113-6.

681 59. Beck, J.R. et al. (2010) A novel family of Toxoplasma IMC proteins displays a hierarchical 682 organization and functions in coordinating parasite division. PLoS Pathog 6 (9), e1001094.

683 60. Frénal, K. et al. (2017) Gliding motility powers invasion and egress in Apicomplexa. Nat Rev 684 Microbiol 15, 645-660.

685 61. Colombo, S. et al. (2005) N-myristoylation determines dual targeting of mammalian NADH686 cytochrome b5 reductase to ER and mitochondrial outer membranes by a mechanism of kinetic 687 partitioning. J Cell Biol 168 (5), 735-45.

688 62. Utsumi, T. et al. (2018) Identification and characterization of protein N-myristoylation 689 occurring on four human mitochondrial proteins, SAMM50, TOMM40, MIC19, and MIC25. PLoS 690 One $13(11)$, e0206355.

691 63. Araiso, Y. et al. (2019) Structure of the mitochondrial import gate reveals distinct preprotein 692 paths. Nature 575 (7782), 395-401.

693 64. Schorr, S. and van der Laan, M. (2018) Integrative functions of the mitochondrial contact site 694 and cristae organizing system. Semin Cell Dev Biol 76, 191-200. 
695 65. van der Laan, M. et al. (2016) Mitochondrial contact site and cristae organizing system. Curr 696 Opin Cell Biol 41, 33-42.

697 66. Ueda, E. et al. (2019) Myristoyl group-aided protein import into the mitochondrial 698 intermembrane space. Sci Rep 9 (1), 1185.

699 67. Humphries, A.D. et al. (2005) Dissection of the mitochondrial import and assembly pathway 700 for human Tom40. J Biol Chem 280 (12), 11535-43.

701 68. Angebault, C. et al. (2018) ER-mitochondria cross-talk is regulated by the Ca(2+) sensor NCS1 702 and is impaired in Wolfram syndrome. Sci Signal 11 (553).

703 69. Baptista, C.G. et al. (2019) Toxoplasma F-box protein 1 is required for daughter cell scaffold 704 function during parasite replication. PLoS Pathog 15 (7), e1007946.

705 70. Thinon, E. et al. (2016) N-Myristoyltransferase Inhibition Induces ER-Stress, Cell Cycle Arrest, 706 and Apoptosis in Cancer Cells. ACS Chem Biol 11 (8), 2165-2176.

707 71. Zha, J. et al. (2000) Posttranslational N-myristoylation of BID as a molecular switch for 708 targeting mitochondria and apoptosis. Science 290 (5497), 1761-1765.

709 72. Utsumi, T. et al. (2003) C-terminal $15 \mathrm{kDa}$ fragment of cytoskeletal actin is posttranslationally $710 \mathrm{~N}$-myristoylated upon caspase-mediated cleavage and targeted to mitochondria. FEBS Lett 539 711 (1-3), 37-44.

712 73. Martin, D.D. et al. (2014) Identification of a post-translationally myristoylated autophagy713 inducing domain released by caspase cleavage of huntingtin. Hum Mol Genet 23 (12), 3166-79. 
714 74. Sakurai, N. and Utsumi, T. (2006) Posttranslational N-myristoylation is required for the anti-

715 apoptotic activity of human tGelsolin, the C-terminal caspase cleavage product of human gelsolin.

716 J Biol Chem 281 (20), 14288-95.

717 75. Vilas, G.L. et al. (2006) Posttranslational myristoylation of caspase-activated p21-activated

718 protein kinase 2 (PAK2) potentiates late apoptotic events. Proc Natl Acad Sci USA 103 (17), 6542-

7196547.

720 76. Martin, D.D. et al. (2012) Tandem reporter assay for myristoylated proteins post-

721 translationally (TRAMPP) identifies novel substrates for post-translational myristoylation:

722 PKCepsilon, a case study. FASEB J 26 (1), 13-28.

723 77. Tapodi, A. et al. (2019) BFSP1 C-terminal domains released by post-translational processing

724 events can alter significantly the calcium regulation of AQP0 water permeability. Exp Eye Res 185,

725107585.

726 78. Bersuker, K. et al. (2019) The CoQ oxidoreductase FSP1 acts parallel to GPX4 to inhibit

727 ferroptosis. Nature 575 (7784), 688-692.

728 79. Doll, S. et al. (2019) FSP1 is a glutathione-independent ferroptosis suppressor. Nature 575

729 (7784), 693-698.

730 80. Conrad, M. et al. (2018) Regulation of lipid peroxidation and ferroptosis in diverse species.

731 Genes \& Development 32 (9-10), 602-619. 
732 81. Timms, R.T. et al. (2019) A glycine-specific N-degron pathway mediates the quality control of

733 protein N-myristoylation. Science 365 (6448), eaaw4912.

734 82. Jiang, H. et al. (2018) Protein lipidation: occurrence, mechanisms, biological Functions, and 735 enabling technologies. Chem Rev 118 (3), 919-988.

736 83. Cao, J. et al. (2019) HDAC11 regulates type I interferon signaling through defatty-acylation of

737 SHMT2. Proc Natl Acad Sci U S A 116 (12), 5487-5492.

738 84. Dian, C. et al. (2020) High-resolution snapshots of human N-myristoyltransferase in action 739 illuminate a mechanism promoting N-terminal Lys and Gly myristoylation. Nat Commun 11 (1), 7401132.

741 85. Kosciuk, T. et al. (2020) NMT1 and NMT2 are lysine myristoyltransferases regulating the ARF6

742 GTPase cycle. Nat Commun $11(1), 1067$.

743 86. Burnaevskiy, N. et al. (2013) Proteolytic elimination of N-myristoyl modifications by the

744 Shigella virulence factor IpaJ. Nature 496 (7443), 106-9.

745 87. Burnaevskiy, N. et al. (2015) Myristoylome profiling reveals a concerted mechanism of ARF

746 GTPase deacylation by the bacterial protease IpaJ. Mol Cell 58 (1), 110-22.

747 88. Belda-Palazon, B. et al. (2019) ABA inhibits myristoylation and induces shuttling of the RGLG1

748 E3 ligase to promote nuclear degradation of PP2CA. Plant J 98 (5), 813-825.

749 89. Kim, S. et al. (2017) Myristoylation of Src kinase mediates Src-induced and high-fat diet750 accelerated prostate tumor progression in mice. J Biol Chem 292 (45), 18422-18433. 
751 90. Colaert, N. et al. (2009) Improved visualization of protein consensus sequences by iceLogo.

$752 \quad$ Nat Methods $6(11), 786-7$.

753 91. Deng, Y. et al. (2013) H+-type and OH- -type biological protonic semiconductors and 754 complementary devices. Sci Rep 3, 2481.

755 92. Maurer-Stroh, S. et al. (2002) N-terminal N-myristoylation of proteins: prediction of substrate

756 proteins from amino acid sequence. J Mol Biol 317 (4), 541-57.

757 93. Alonso, A.M. et al. (2019) Exploring protein myristoylation in Toxoplasma gondii. Exp Parasitol $758 \quad 203,8-18$.

759 


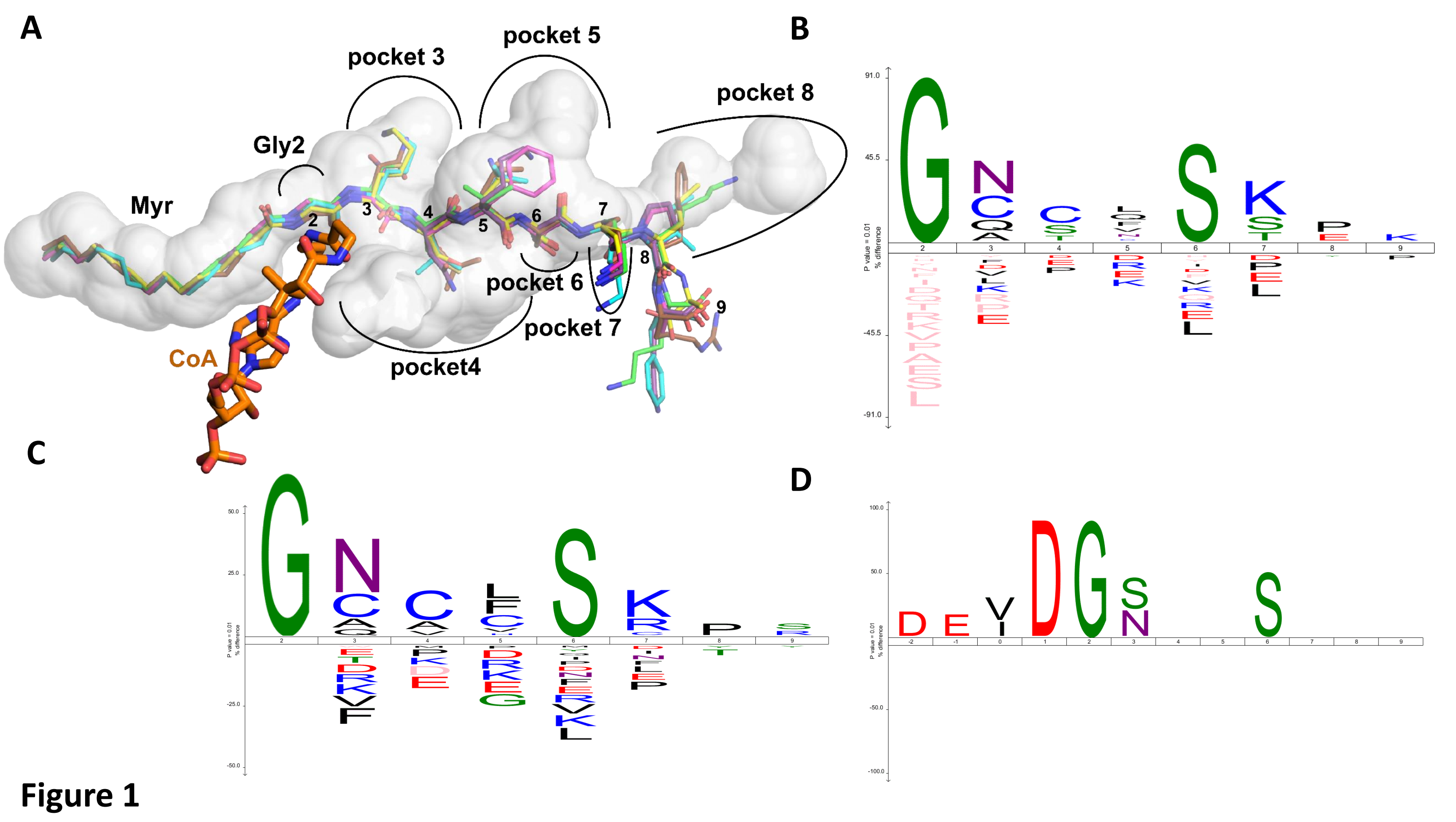


Surface Y356 W12 W11 W10 W9 W8 W7 W6 W5 W4 W2 w3 Catalytic center

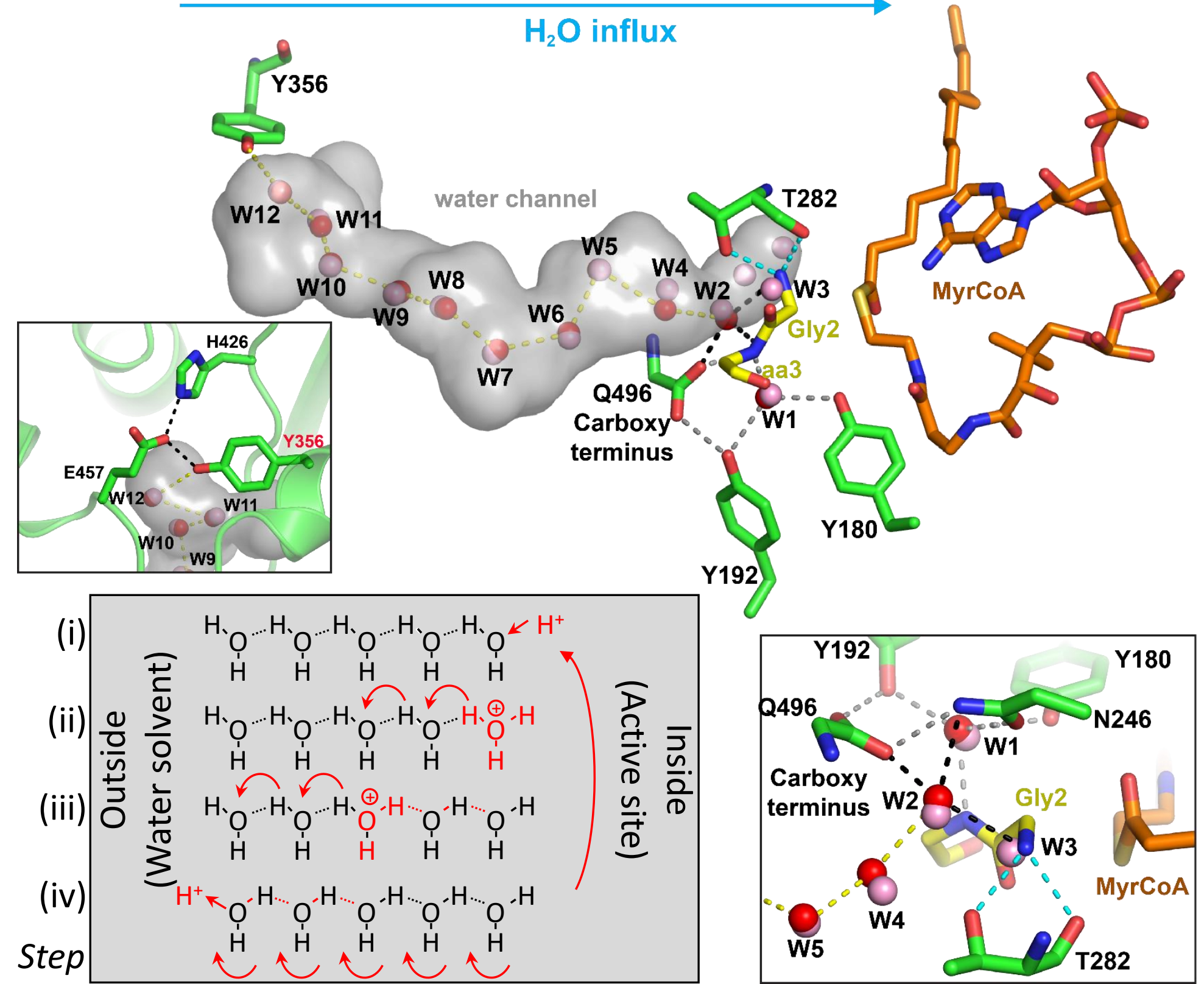

Figure 2 


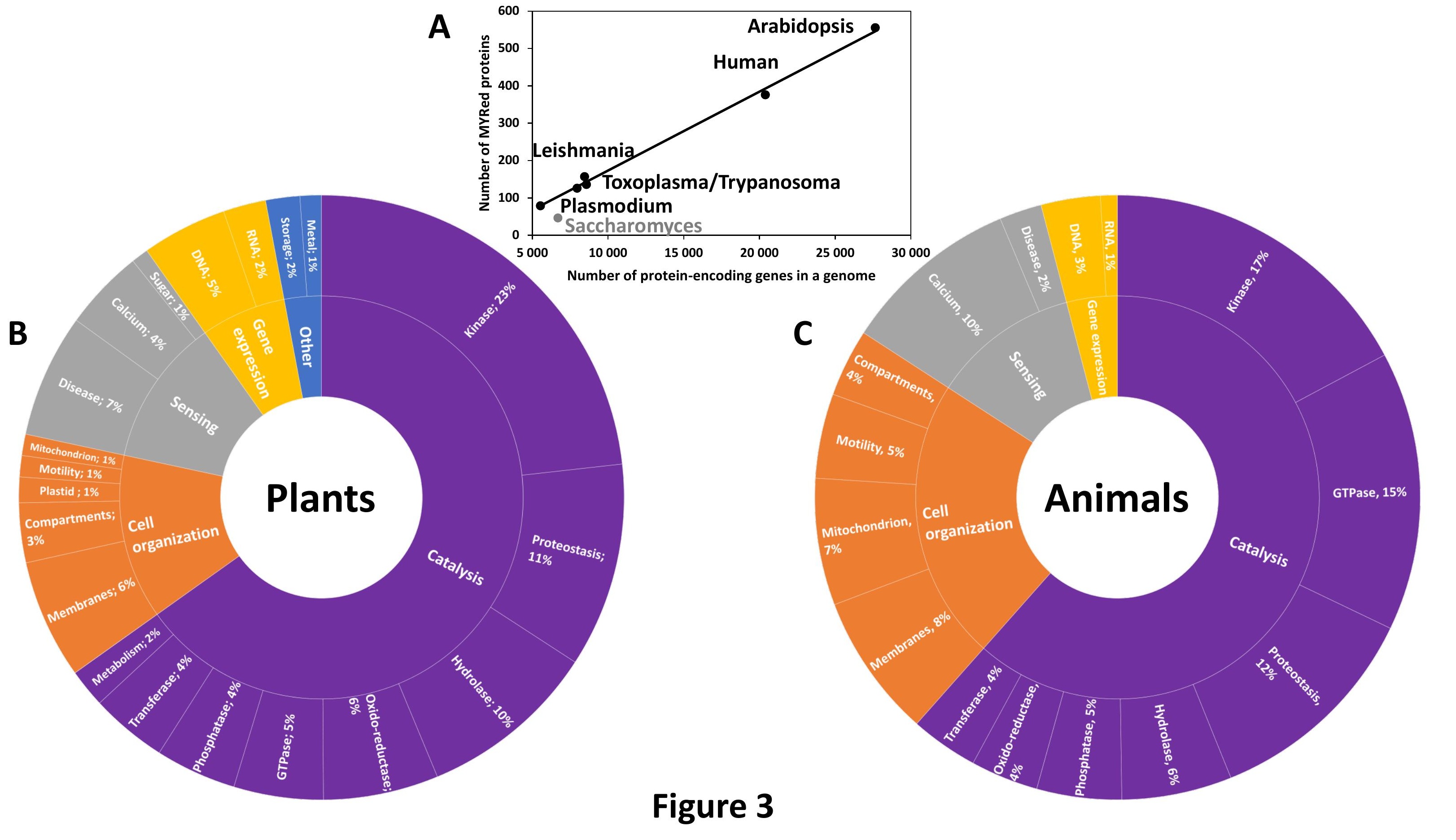




\section{Box 1}

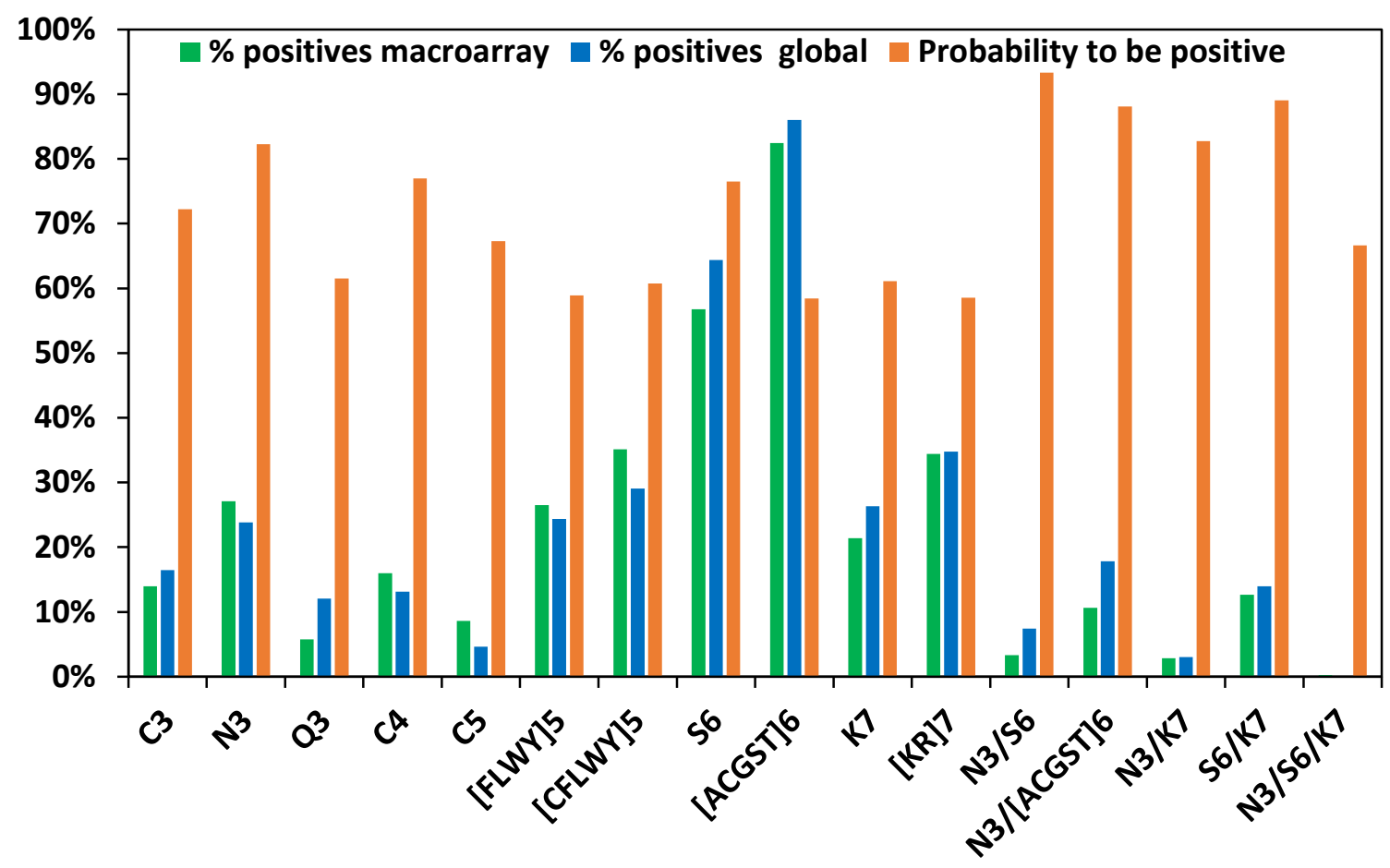

Figure I 